Document downloaded from:

http://hdl.handle.net/10251/83649

This paper must be cited as:

Rueda Segado, MP.; Sánchez Pérez, EA. (2015). Compactness in spaces of p-integrable functions with respect to a vector measure. Topological Methods in Nonlinear Analysis. 45(2):641-653. doi:10.12775/TMNA.2015.030.

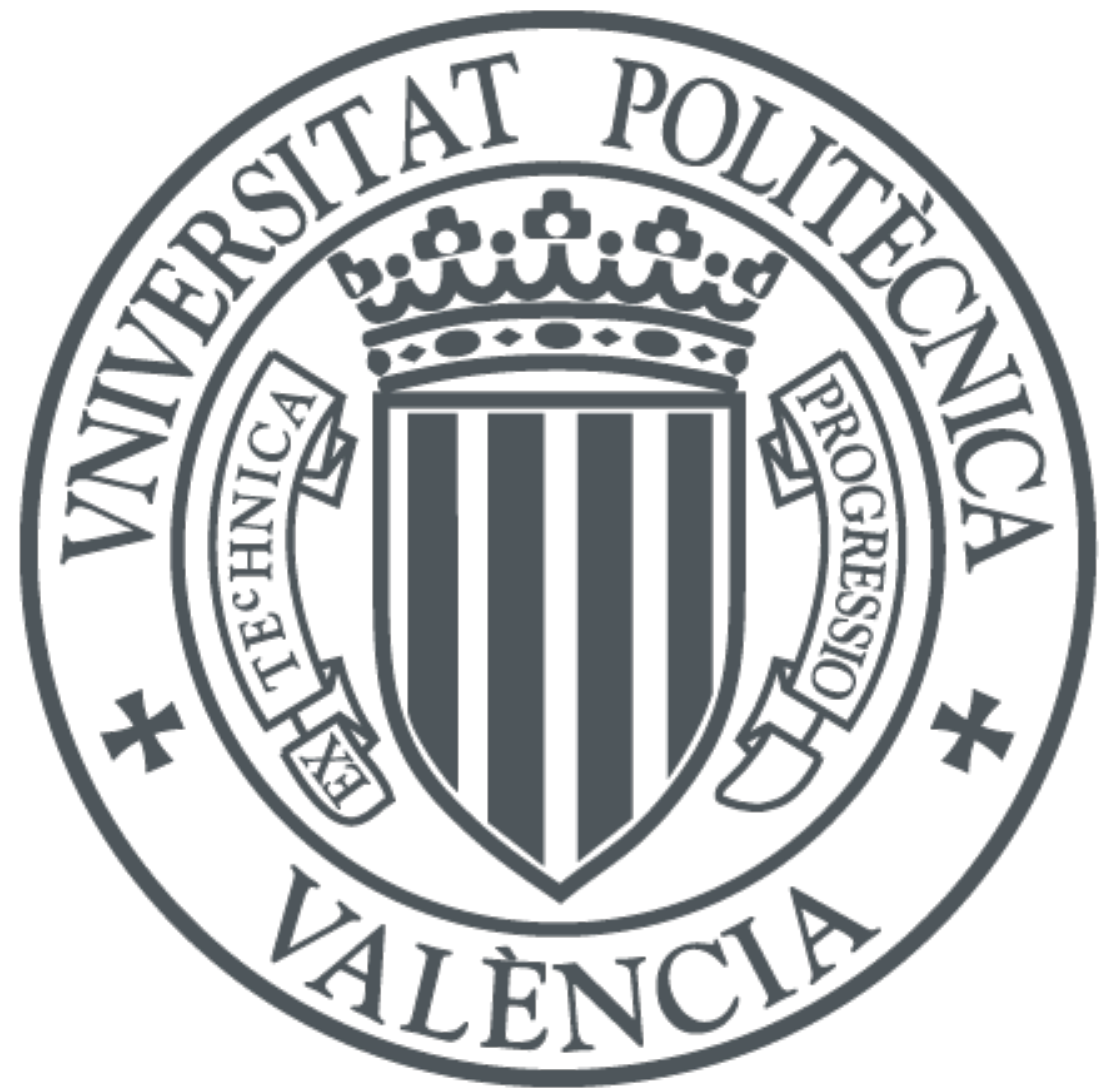

The final publication is available at

http://dx.doi.org/10.12775/TMNA.2015.030

Copyright JULIUSZ SCHAUDER CTR NONLINEAR STUDIES

Additional Information

"The final publication is available at the TMNA webpage via http://dx.doi.org/10.12775/TMNA.2015.030". 


\title{
COMPACTNESS IN SPACES OF $p$-INTEGRABLE FUNCTIONS WITH RESPECT TO A VECTOR MEASURE.
}

\author{
P. RUEDA AND E.A. SÁNCHEZ PÉREZ
}

\begin{abstract}
We prove that, under some reasonable requirements, the unit balls of the spaces $L^{p}(m)$ and $L^{\infty}(m)$ of a vector measure of compact range $m$ are compact with respect to the topology $\tau_{m}$ of pointwise convergence of the integrals. This result can be considered as a generalization of the classical Alaoglu Theorem to spaces of $p$-integrable functions with respect to vector measures with relatively compact range. Some applications to the analysis of the Saks spaces defined by the norm topology and $\tau_{m}$ are given.
\end{abstract}

\section{INTRODUCTION}

The origin of the integration theory with respect to vector measures goes back to control theory. The solutions of some important problems in this area have been obtained using vector measure tools (see [17]). Since then, the interest of the theory has yielded to the development of the theory of $L^{p}(m)$ spaces with respect to a vector measure $m$ and has found more applications in other areas as non linear analysis of signals (see $[12,14,16])$. The techniques coming from vector measure integration have also been successfully applied in mathematical physics to solve some classical non linear problems (see $[4,11,15]$ ).

2010 Mathematics Subject Classification. 46A50, 46B50, 46E30, 46G10.

Key words and phrases. Banach function space, vector measure integration, compactness.

P. Rueda acknowledges with thanks the support of the Ministerio de Economía y Competitividad (Spain) MTM2011-22417. E.A. Sánchez Pérez acknowledges with thanks the support of the Ministerio de Economía y Competitividad (Spain) MTM2012-36740-C02-02. 
It is usual that, when extending a theory to a wider context, some of the properties that were satisfied in the restricted theory are not longer valid in the more general situation. This is the case when one goes from the integration theory with respect to scalar measures to integration theory with respect to vector measures. In this case, the role played by the weak* topology is assumed by the topology $\tau_{m}$ of the pointwise convergence of the integrals. However, it is not true in general that the closed unit ball of the space $L^{p}(m)$ of all $p$-integrable functions with respect to a (countably additive) vector measure $m$ on the measure space $(\Omega, \Sigma)$ and with values in a Banach space $E$, must be $\tau_{m}$-compact. This is so since in general the dual of $L^{p}(m)$ cannot be identified with $L^{p^{\prime}}(m)$, where $\frac{1}{p}+\frac{1}{p^{\prime}}=1,1 \leq p \leq \infty$. The relation between the convergence of sequences in the spaces of vector measure integrable functions and the convergence of the corresponding vector valued integrals has been studied since the seventies (see for instance [18, 19], [5, Section 6], [26] and the references therein) and yields to replacing the duality of the Banach space $L^{p}(m)$ by the vector measure duality, i.e the duality induced between $L^{p}(m)$ and $L^{p^{\prime}}(m)$ by the vector valued integration. This duality has to be understood in the following sense. The vector measure integral defines an integration operator $I_{m}$ from the space $L^{1}(m)$ of integrable functions with respect to the vector measure $m$ and the Banach space $E$. Then the map $(f, g) \rightsquigarrow \int_{\Omega} f g d m \in E$ defines a bilinear operator $I_{m}(\cdot, \cdot): L^{p}(m) \times L^{p^{\prime}}(m) \rightarrow E$ that satisfies the requirements for defining a useful duality between both spaces. This vector measure duality yields to consider the topology $\tau_{m}$ on $L^{p}(m)$, given by the pointwise convergence of the integrals $\int_{\Omega} \cdot g d m, g \in L^{p^{\prime}}(m)$. For $1<p<\infty$, the $\tau_{m}$ topology lies between the weak and the norm topologies on (norm) bounded sets and the problem of establishing 
conditions on compactness for $\tau_{m}$ (besides the straightforward ones) is in order.

It is well known that compactness is a powerful tool in Functional Analysis and, in particular, in Vector Measure Theory. For instance, it was already shown in [28] that compactness of the unit ball of the spaces $L^{p}(m)$ with respect to $\tau_{m}$ was necessary for the main factorization theorems of operators to work successfully, but no explicit results of when this property holds were shown. More efforts have been made in this direction since 2000, sometimes by analyzing compactness properties of the integration map, that is closely related to the quoted problem (see for example $[9,13,24]$ and [26, Ch.3] and the references therein). A relevant paper where the relations between the weak topology on $L^{p}(m), 1<p<\infty$, and the weak integrals defined by the integration map is [9], where it is shown that the weak topology and this topology defined by the weak integrals $\int_{\Omega} \cdot g d\left\langle m, x^{*}\right\rangle, g \in L^{p^{\prime}}(m), x^{*} \in X^{*}$, coincide on bounded sets. In this direction, the papers [7, 29] show descriptions of the dual space of $L^{p}(m), 1<p<\infty$ by characterizing the topology defined by the weak integrals.

The aim of this paper is to analyze some aspects of the $\tau_{m}$ topology and mainly to provide some criteria for $\tau_{m}$-compactness of the unit balls of $L^{p}(m)$ and $L^{\infty}(m)$. Some examples illustrating our results are also shown. It must be said that the spaces $L^{p}(m)$ represent in fact the class of all order continuous p-convex Banach lattices with a weak unit (see [6, Prop.2.4] or [26, Prop.3.30]), what means that our results can be applied to a broad class of Banach spaces.

The paper is organized as follows. After a preliminary section with definitions and notation, in Section 3 we analyze compactness of the closed unit ball of $L^{p}(m)$ with respect to $\tau_{m}$ and so, to all the natural topologies on $L^{p}(m)$. Among others, we prove the following result 
(Corollary 8): If $1<p<\infty, L^{p}(m)$ is reflexive, $\left(B_{L^{p^{\prime}}(m)},\|\cdot\|_{L^{p^{\prime}}(m)}\right)$ is separable and $m$ has relatively compact range, then $\left(B_{L^{p}(m)}, \tau_{m}\right)$ is compact. Our main result is a generalization of Alaoglu's Theorem to spaces of $p$-integrable functions with respect to vector measures with relatively compact range with respect to $\tau_{m}$ (see Theorem 10). In Section 4 we give some applications to the Saks space structure of the $L^{p}(m)$-spaces.

\section{Preliminaries}

Our notation is standard. If $1 \leq p \leq \infty$, we write $p^{\prime}$ for the extended real number satisfying $1 / p+1 / p^{\prime}=1$. Let $E$ be a real Banach space and let $(\Omega, \Sigma)$ be a measurable space. If $\mu$ is a finite measure on $(\Omega, \Sigma)$ we define Banach function space over $\mu$ following [20, Def.1.b.17, p.28]; it is a Banach space given by an ideal of $L^{1}(\mu)$ containing simple functions with a lattice norm. Given a countably additive vector measure $m$ : $\Sigma \rightarrow E$, we write $\mathcal{R}(m)$ for its range. Its variation $|m|$ is defined by

$$
|m|(A):=\sup _{B_{i} \in \pi} \sum_{i=1}^{n}\left\|m\left(B_{i}\right)\right\|,
$$

where the supremum is computed over all finite measurable partitions $\pi$ of $A \in \Sigma$. We write $\|m\|$ for its semivariation, that is defined by $\|m\|(A):=\sup _{x^{*} \in B_{E^{*}}}\left|\left\langle m, x^{*}\right\rangle\right|(A), A \in \Sigma$, where $\left\langle m, x^{*}\right\rangle$ is the scalar measure given by $\left\langle m, x^{*}\right\rangle(A):=\left\langle m(A), x^{*}\right\rangle$. It is known that there exists $x^{*} \in E^{*}$ such that $m$ is absolutely continuous with respect to $\left|\left\langle m, x^{*}\right\rangle\right|$, that is $m(A)=0$ whenever $\left|\left\langle m(A), x^{*}\right\rangle\right|=0$. Such a measure $\left\langle m, x^{*}\right\rangle$ is called a Rybakov measure for $m$ (see [3, Ch.IX]). Throughout the paper $\mu$ will denote a fixed Rybakov measure for $m$. If $1 \leq p<$ $\infty$, a (scalar) measurable function $f$ is said to be $p$-integrable with respect to $m$ if $|f|^{p}$ is integrable with respect to all measures $\left|\left\langle m, x^{*}\right\rangle\right|$ and for each $A \in \Sigma$ there exists an element $\int_{A}|f|^{p} d m \in E$ such that 
$\left\langle\int_{A}|f|^{p} d m, x^{*}\right\rangle=\int_{A}|f|^{p} d\left\langle m, x^{*}\right\rangle, x^{*} \in E^{*}$ (see [18, 19] or [26, Ch.3]). For the case $p=\infty, L^{p}(m)$ is simple defined as $L^{\infty}(\mu)$ for a Rybakov measure $\mu$ for $m$ (obviously all Rybakov measures give the same space).

The space $L^{p}(m), 1 \leq p<\infty$, is defined by all ( $\mu$-equivalence classes of) measurable real functions defined on $\Omega$ that are $p$-integrable with respect to $m$. It is a Banach function space over any Rybakov measure for $m$ when the a.e. order and the norm

$$
\|f\|_{L^{p}(m)}:=\left(\sup _{x^{*} \in B_{E^{*}}} \int_{\Omega}|f|^{p} d\left|\left\langle m, x^{*}\right\rangle\right|\right)^{1 / p}, \quad f \in L^{p}(m),
$$

are considered. It is an order continuous $p$-convex —with constant 1 - Banach function space over each Rybakov measure for $m$ (see [28, Proposition 5], and [6] and [26, Ch.3] for more information on these spaces). An important fact is that $f g \in L^{1}(m)$ whenever $f \in L^{p}(m)$ and $g \in L^{p^{\prime}}(m)$, for each $1 \leq p \leq \infty$ (see [26, Prop.3.43] and [28, Sec.3]; see also [6]). Moreover, for each $f \in L^{p}(m)$

$$
\|f\|_{L^{p}(m)}=\sup _{g \in B_{L_{p^{\prime}}(m)}}\left\|\int_{\Omega} f g d m\right\| .
$$

Fix a Rybakov measure $\mu$ for $m$. Due to the order continuity of $L^{p}(m)$, its dual space $L^{p}(m)^{*}(1 \leq p<\infty)$ allows an easy description; it coincides with its Köthe dual (or associate space) $\left(L^{p}(m)\right)^{\prime}$, that is, $L^{p}(m)^{*}=\left(L^{p}(m)\right)^{\prime}=\left\{\varphi_{g}: g \in \mathcal{H}\right\}$, where

$$
\mathcal{H}:=\left\{g: \Omega \rightarrow \mathbb{R} \quad \Sigma-\text { measurable }: f g \in L^{1}(\mu) \text { for all } f \in L^{p}(m)\right\}
$$

and the duality is given by $\left\langle\varphi_{g}, f\right\rangle=\int_{\Omega} f g d \mu$. Information about a precise description of $\left(L^{p}(m)\right)^{\prime}$ can be found in $[7,8,9,10,29]$. The integration operator $I_{m}: L^{1}(m) \rightarrow E$ is given by

$$
I_{m}(f)=\int_{\Omega} f d m, f \in L^{1}(m) .
$$

General information on the properties of $I_{m}$ can be found in $[23,24,25]$, and [26] and the references therein. Since for all $p>1$ the inclusion $L^{p}(m) \subseteq L^{1}(m)$ always holds, the integration operator can be defined 
also as an operator $I_{m}: L^{p}(m) \rightarrow E$. If $f \in L^{1}(m)$, the set function $m_{f}: \Sigma \rightarrow E$ defines a vector measure too, and so the integration map $I_{m_{f}}: L^{1}\left(m_{f}\right) \rightarrow E$ can be considered.

Let $1 \leq p \leq \infty$. In this paper we will consider the following locally convex topologies on $L^{p}(m)$.

- The topology induced by the norm $\|\cdot\|_{L^{p}(m)}$.

- The topology of the pointwise convergence of the integrals $\tau_{m}$, i.e. the topology that is defined by the seminorms $\gamma_{g}(f):=\left\|\int_{\Omega} f g d m\right\|$, $f \in L^{p}(m), g \in L^{p^{\prime}}(m)$.

- The topology of the pointwise weak convergence of the integrals $\tau_{w, m}$, that is given by the seminorms $\gamma_{g, x^{*}}(f):=\left\langle\int_{\Omega} f g d m, x^{*}\right\rangle, f \in$ $L^{p}(m), g \in L^{p^{\prime}}(m), x^{*} \in E^{*}$. Is is a locally convex (and so Hausdorff) topology on $L^{p}(m)$.

- The weak topology $\tau_{w}$ on $L^{p}(m)$.

Clearly, the norm topology is finer than all the others, and $\tau_{m}$ and $\tau_{w}$ are finer than $\tau_{w, m}$. However, in general $\tau_{m}$ is not finer than $\tau_{w}$. Indeed, $\tau_{m}$ coincides with the weak* topology whenever $m$ is a scalar measure and for $p=1$, the weak and the weak* topologies do not coincide.

As we said in the Introduction, we are mainly interested in the space $\left(L^{p}(m), \tau_{m}\right)$. All the basic results we will need on its topological properties are explained through the paper, and can be found in $[6,8,9,26,28]$.

\section{3. $\tau_{m}$-COMPACTNESS OF THE UNIT BALLS OF $L^{p}(m)$ AND $L^{\infty}(m)$}

In this section we obtain characterizations of the $\tau_{m}$-compactness of $B_{L^{p}(m)}$ and analyze several consequences. The coincidence of the weak topology and $\tau_{w, m}$ on bounded subsets of $L^{p}(m)$ was shown in [9, Theorem 3.5] for $1<p<\infty$ (see also [8]). So, $\left(L^{p}(m),\|\|_{L^{p}(m)}\right)$ is reflexive if and only if $B_{L^{p}(m)}$ is $\tau_{w, m}$-compact. 
First of all, the range of $m$ to be relatively compact is clearly a necessary requirement for the $\tau_{m}$ compactness of the unit ball. This is so because if $B_{L^{p}(m)}$ is $\tau_{m}$ compact, $p>1$, then its image $I_{m}\left(B_{L^{p}(m)}\right)$ is compact, where $I_{m}: L^{p}(m) \rightarrow E$ is the integration map, and since the range of $m$ is included in $K I_{m}\left(B_{L^{p}(m)}\right)$ for some $K>0$, we get that it is also relatively compact. However, this condition by itself does not imply that $I_{m}: L^{1}(m) \rightarrow E$ is compact: there is a broad class of cases for which the range of $m$ is compact but $I_{m}: L^{1}(m) \rightarrow E$ is not compact (see [26, Sec.3.3]).

It is worth mentioning that if $I_{m}: L^{1}(m) \rightarrow E$ is compact, then $L^{1}(m)$ is in fact order isomorphic to $L^{1}(|m|$ ) (a scalar measure, see Proposition 3.48 in [26]), and so the $\tau_{m}$ topology is just given by the weak* topology: nothing new is provided.

Proposition 1. Let $1 \leq p \leq \infty$. If $B_{L^{p}(m)}$ is $\tau_{m}$-compact, then $\tau_{w, m}$ and $\tau_{m}$ coincide on bounded subsets of $L^{p}(m)$. Moreover, if $p>1$ and $B_{L^{p}(m)}$ is $\tau_{m}$-compact, then the weak topology and $\tau_{m}$ coincide on bounded subsets of $L^{p}(m)$. Consequently, if $p>1, B_{L^{p}(m)}$ is $\tau_{m^{-}}$ compact if and only if $\left(L^{p}(m),\|\cdot\|_{L^{p}(m)}\right)$ is reflexive and the weak topology and $\tau_{m}$ coincide on $B_{L^{p}(m)}$.

Proof. Consider the identity map $I:\left(B_{L^{p}(m)}, \tau_{m}\right) \rightarrow\left(B_{L^{p}(m)}, \tau_{w, m}\right)$. As $I$ is continuous, bijective and $B_{L^{p}(m)}$ is $\tau_{m}$-compact, then $I$ is an homeomorphism. Hence the weak topology and $\tau_{m}$ coincide on $B_{L^{p}(m)}$. The result for the weak topology is a consequence of the coincidence between this topology and $\tau_{w, m}$.

Compactness of $\left(B_{L^{p}(m)}, \tau_{m}\right)$ allows also to give a description of $L^{p}(m)$ as a dual space. Define

$$
Z=\left\{T \in L^{p}(m)^{*}:\left.T\right|_{B_{L^{p}(m)}} \text { is } \tau_{m}-\text { continuous }\right\}
$$

endowed with the topology induced by the strong topology on $L^{p}(m)^{*}$. 
Proposition 2. Let $1<p<\infty$. If $B_{L^{p}(m)}$ is $\tau_{m}$-compact, then the space $\left(L^{p}(m),\|\|_{L^{p}(m)}\right)$ is isometrically isomorphic to the dual space $Z^{*}$ of $Z$.

Proof. It follows from [22, Theorem 1].

For each $f \in L^{p}(m)$ consider the integration operator $I_{m, f}: L^{p^{\prime}}(m) \rightarrow$ $E$ given by $I_{m, f}(h)=\int_{\Omega} f h d m$. We can identify each $f$ with $I_{m, f}$ and then consider $L^{p}(m)$ as a subspace of the product $E^{L^{p^{\prime}}(m)}$. Using the equivalent expression for the norm given in (1), it is easy to see that the product topology coincides on $L^{p}(m)$ with $\tau_{m}$. In particular, a sequence $\left(g_{n}\right)_{n}$ in $L^{p}(m)$ converges for $\tau_{m}$ if and only if $\left(I_{m, g_{n}}\right)_{n}$ converges pointwise. Recall that if $m: \Sigma \rightarrow E$ is a vector measure, then the space $L^{p}(m)$ is weakly sequentially complete whenever $E$ is (see [6, Proposition 2.2]; see also Proposition 2.7 in this paper). Sequential completeness of the space $\left(L^{p}(m), \tau_{m}\right)$ becomes a relevant tool in this paper.

Theorem 3. Let $1 \leq p<\infty$. If $\left(B_{L^{p}(m)}, \tau_{w, m}\right)$ is sequentially complete, then $\left(B_{L^{p}(m)}, \tau_{m}\right)$ is sequentially complete.

Proof. Let $\left(g_{n}\right)_{n}$ be a $\tau_{m}$-Cauchy sequence in $B_{L^{p}(m)}$. Since $\tau_{w, m}$ is coarser than $\tau_{m}$, the sequence $\left(g_{n}\right)_{n}$ is $\tau_{w, m}$ Cauchy. By the assumption that $B_{L^{p}(m)}$ is $\tau_{w, m}$ sequentially complete, $\left(g_{n}\right)_{n} \tau_{w, m^{-}}$-converges to an element $g \in B_{L^{p}(m)}$. Let us prove that $\left(g_{n}\right)_{n}$ actually converges to $g$ for $\tau_{m}$ or, equivalently, that $\left(I_{m, g_{n}}\right)_{n}$ converges pointwise to $I_{m, g}$. Since $E^{L^{p^{\prime}}(m)}$ is complete and $\left(I_{m, g_{n}}\right)_{n}$ is Cauchy for the product topology, $\left(I_{m, g_{n}}\right)_{n}$ converges pointwise to some $T \in E^{L^{p^{\prime}}(m)}$. It suffices to prove that $T=I_{m, g}$. Given arbitrary $h \in L^{p^{\prime}}(m)$ and $x^{*} \in E^{*}$, consider the functional $\gamma_{h, x^{*}} \in\left(L^{p}(m)\right)^{\prime}$ given by $\gamma_{h, x^{*}}(f)=\left\langle\int_{\Omega} f h d m, x^{*}\right\rangle$, 
$f \in L^{p}(m)$. As $\left(g_{n}\right)_{n} \tau_{w, m}$-converges to $g$, then

$$
\begin{aligned}
\left\langle I_{m, g}(h), x^{*}\right\rangle & =\left(\gamma_{h, x^{*}}\right)(g)=\lim _{n}\left(\gamma_{h, x^{*}}\right)\left(g_{n}\right) \\
& =\lim _{n}\left\langle I_{m, g_{n}}(h), x^{*}\right\rangle=\left\langle T(h), x^{*}\right\rangle,
\end{aligned}
$$

that allows to conclude that $I_{m, g}=T$.

Note that the proof works with the obvious changes to prove that $\left(L^{p}(m), \tau_{m}\right)$ is sequentially complete whenever $\left(L^{p}(m), \tau_{w, m}\right)$ is sequentially complete, $1 \leq p<\infty$.

Lemma 4. [8, Proposition 2.2.1] Let $1 \leq p<\infty$. If $\left(B_{L^{p^{\prime}(m)}},\|\cdot\|_{L^{p^{\prime}}(m)}\right)$ is separable, then $\left(B_{L^{p}(m)}, \tau_{m}\right)$ is metrizable.

Proof. Take $\left(g_{n}\right)_{n}$ a norm dense sequence in $B_{L^{p^{\prime}}(m)}$. Since the integration operator $I_{m, f}: L^{p^{\prime}}(m) \rightarrow E$ is continuous for all $f \in L^{p}(m)$ and $\left(g_{n}\right)_{n}$ is dense, the map $J: B_{L^{p}(m)} \rightarrow B_{E}^{\mathbb{N}}$ given by $J(f)=\left(\int_{\Omega} g_{n} f d m\right)_{n}$ is injective. Since $\tau_{m}$ coincides with the product topology restricted to $B_{L^{p}(m)}$ and $B_{E}^{\mathbb{N}}$ is metrizable, we conclude that $\left(B_{L^{p}(m)}, \tau_{m}\right)$ is metrizable.

The reader is referred to [8, Section 2.2] for related results on separability and metrizability. We recall that a locally convex space is quasi-complete if every closed bounded subset is complete.

Corollary 5. Let $1 \leq p<\infty$. If $\left(B_{L^{p}(m)}, \tau_{w, m}\right)$ is sequentially complete and $\left(B_{L^{p^{\prime}(m)}},\|\cdot\|_{L^{p^{\prime}(m)}}\right)$ is separable, then $\left(B_{L^{p}(m)}, \tau_{m}\right)$ is complete.

Proof. By Lemma $4 B_{L^{p}(m)}$ is $\tau_{m^{-}}$-metrizable. So, to see that $B_{L^{p}(m)}$ is $\tau_{m}$-complete it suffices to be proved that $B_{L^{p}(m)}$ is $\tau_{m^{-}}$-sequentially complete. This follows from Theorem 3.

Remark 6. Every $\tau_{m}$-bounded set $A$ in $L^{p}(m)$ is $\|\cdot\|_{L^{p}(m)}$-bounded. Indeed, the set of operators $\left\{I_{m, f}: f \in A\right\}$ is pointwise bounded, for

$$
\sup _{f \in A}\left\|I_{m, f}(g)\right\|=\sup _{f \in A}\left\|\int_{\Omega} f g d m\right\|=\sup _{f \in A} \gamma_{g}(f)<\infty
$$


for any $g \in L^{p^{\prime}}(m)$. By the Banach Steinhauss theorem of uniform boundedness,

$$
\sup _{f \in A}\left\|I_{m, f}\right\|=\sup _{f \in A} \sup _{g \in B_{L^{p^{\prime}(m)}}}\left\|\int_{\Omega} f g d m\right\|=\sup _{f \in A}\|f\|_{L^{p}(m)}<\infty .
$$

Hence, $A$ is $\|\cdot\|_{L^{p}(m)}$-bounded. On the other hand, $B_{L^{p}(m)}$ is $\tau_{m}$ closed (see [28, Proposition 12]) and bounded. Therefore, $\left(B_{L^{p}(m)}, \tau_{m}\right)$ is complete if and only if $\left(L^{p}(m), \tau_{m}\right)$ is quasi-complete.

We will denote by $\mathcal{R}(m)$ the range of $m$. We now proceed to give conditions that ensure compactness of $\left(B_{L^{p}(m)}, \tau_{m}\right)$.

Theorem 7. Let $1<p \leq \infty$ and let $m$ be a vector measure such that $\left(B_{L^{p}(m)}, \tau_{m}\right)$ is complete. If $\mathcal{R}(m)$ is relatively compact, then $\left(B_{L^{p}(m)}, \tau_{m}\right)$ is compact. If $p=1$, the result remains true under the assumption that $I_{m}: L^{1}(m) \rightarrow E$ is compact.

Proof. Let $1<p \leq \infty$. As in the previous proofs, consider $B_{L^{p}(m)}$ as a subspace of $E^{B_{L^{p^{\prime}}(m)}}$ by means of the injection $f \mapsto\left(\int_{\Omega} g f d m\right)_{g \in B_{L^{p^{\prime}}(m)}}$. For each $g \in B_{L^{p^{\prime}(m)}}$, let $\left.K_{g}:=\overline{I_{m, g}\left(B_{L_{p}}(m)\right.}\right)$. Since $\mathcal{R}(m)$ is relatively compact, then the integration map $I_{m}: L^{p}(m) \rightarrow E$ is compact (see Proposition 3.56 in [26]). Hence, each $I_{m, g}$ is clearly compact when $g$ is a simple function. Taking into account that the norm of the operator $I_{m, g}$ coincides with $\|g\|_{L^{p^{\prime}(m)}}$ and the ideal of compact operators is closed for the operator norm, we obtain that each $K_{g}$ is compact in $E$, for any $g \in B_{L^{p^{\prime}(m)}}$.

By Tychonoff's Theorem, the product $\prod_{g \in B_{L^{p^{\prime}(m)}}} K_{g}$ is compact. Since

$$
B_{L^{p}(m)} \subset \prod_{g \in B_{L^{p^{\prime}(m)}}} I_{m, g}\left(B_{L^{p}(m)}\right) \subset \prod_{g \in B_{L^{p^{\prime}}(m)}} K_{g}
$$

and the product topology coincides with $\tau_{m}$ on $B_{L^{p}(m)}$, it suffices to be proved that $B_{L^{p}(m)}$ is closed in $\prod_{g \in B_{L^{p^{\prime}}(m)}} K_{g}$. This follows from the 
completeness of $\left(B_{L^{p}(m)}, \tau_{m}\right)$ and the coincidence of $\tau_{m}$ and the product topology.

The same argument works for $p=1$ taking into account that $I_{m}$ compact implies that $\mathcal{R}(m)$ and so $I_{m, g}$ is compact for all simple functions $g$, that are dense in $L^{\infty}(m)$.

Corollary 8. Let $1<p<\infty$ and let $m$ be a vector measure such that $L^{p}(m)$ is weakly sequentially complete - equivalently, $L^{p}(m)$ is reflexive-, and $\left(B_{L^{p^{\prime}}(m)},\|\cdot\|_{L^{p^{\prime}}(m)}\right)$ is separable. If $\mathcal{R}(m)$ is relatively compact, then $\left(B_{L^{p}(m)}, \tau_{m}\right)$ is compact. For $p=1$ the result is true under the assumption that $I_{m}: L^{1}(m) \rightarrow E$ is compact.

Proof. For $1<p<\infty$, weak sequential completeness of $L^{p}(m)$ and reflexivity are equivalent properties (see Proposition 3.38 in [26]). Since $L^{p}(m)$ is weakly sequentially complete, $B_{L^{p}(m)}$ so is. Since $\tau_{w}$ and $\tau_{w, m}$ coincide on bounded sets when $1<p<\infty$ [9, Theorem 3.5] it follows that $B_{L^{p}(m)}$ is $\tau_{w, m}$ sequentially complete. By Corollary $5 B_{L^{p}(m)}$ is $\tau_{m}$ complete. Theorem 7 gives now the result.

Remark 9. Proposition 2.3 in [6] provides a characterization for the separability of $L^{p^{\prime}}(m)$. For the weak sequential completeness, see the comments in page 14 of [6] and Proposition 3.38 of [26].

Corollary 8 clarifies Theorem 14 in [28] where a similar result is stated, whose proof assumes implicitly that the unit ball $B_{L^{p}(m)}$ is $\tau_{m^{-}}$ complete.

In particular, Theorem 7 gives sufficient conditions for $B_{L^{\infty}(m)}$ to be $\tau_{m}$-compact. Without assuming that $B_{L^{p^{\prime}(m)}}$ is separable and $L^{p}(m)$ is weakly sequentially complete, a slightly different argument provides the following result on compactness of $B_{L^{\infty}(m)}$ with respect to the topology induced by $\left(L^{p}(m), \tau_{m}\right)$. In what follows we will assume for the aim of simplicity and without loss of generality that $B_{L^{\infty}(m)} \subseteq B_{L^{p}(m)}$, 
or equivalently that $\|m\|(\Omega) \leq 1$. The proof uses a Radon-Nikodym Theorem for vector measures.

Theorem 10. Let $1<p \leq \infty$. Consider a vector measure $m$. If $\mathcal{R}(m)$ is relatively compact, then $B_{L^{\infty}(m)}$ considered as a topological subspace of $\left(B_{L^{p}(m)}, \tau_{m}\right)$, is compact. If $p=1$, the same result holds under the assumption of compactness for $I_{m}: L^{1}(m) \rightarrow E$.

Proof. For each $g \in B_{L^{p^{\prime}(m)}}$ let $\left.K_{g}:=\overline{I_{m, g}\left(B_{L^{p}}(m)\right.}\right)$. Consider now $B_{L^{\infty}(m)} \subset B_{L^{p}(m)}$ contained in the product $\prod_{g \in B_{L^{p^{\prime}(m)}}} K_{g}$ as was explained in the proof of Theorem 7. For $p>1$ each subset $K_{g}$ is compact (see [26, Ch. 3] or [27, Lemma 14]); for $p=1$, since $I_{m}$ is assumed to be compact, the same holds. Take a Cauchy net $\left\{g_{\eta}\right\}_{\eta \in \Lambda}$ in $B_{L^{\infty}(m)}$ for the topology induced by $\left(L^{p}(m), \tau_{m}\right)$. Note that for all $\eta, \Sigma \ni A \rightsquigarrow \int_{A} g_{\eta} d m$ defines a (countably additive) vector measure $m_{g_{\eta}}$. By the Banach-Steinhauss theorem the pointwise limit of $I_{m_{g_{\eta}}}$ is a continuous linear operator $T: L^{p^{\prime}}(m) \rightarrow E$. Define the set function $m_{T}: \Sigma \rightarrow E$ by $m_{T}(A):=T\left(\chi_{A}\right), A \in \Sigma$. Let us consider two cases:

(i) If $1 \leq p^{\prime}<\infty$, then $L^{p^{\prime}}(m)$ is order continuous and so $m_{T}$ is a (countably additive) vector measure. Clearly, the following domination holds: for each $g_{\eta}, A \in \Sigma$ and $x^{\prime} \in B_{X^{*}}$,

$$
\left\langle\int_{A} g_{\eta} d m, x^{\prime}\right\rangle \leq\left\|g_{\eta}\right\|_{L^{\infty}(m)}\left|\left\langle m, x^{\prime}\right\rangle\right|(A) .
$$

In other words, each vector measure given by $I_{m_{g_{\eta}}}$, and so the pointwise limit $T$, is scalarly uniformly dominated by $m$. Indeed, for each $A \in \Sigma$ and $x^{\prime} \in B_{X^{*}},\left\langle T\left(\chi_{A}\right), x^{\prime}\right\rangle=\lim _{\eta}\left\langle\int_{A} g_{\eta} d m, x^{\prime}\right\rangle \leq\left|\left\langle m, x^{\prime}\right\rangle\right|(A)$.

(ii) If $p=1$ (i.e. $p^{\prime}=\infty$ ), although $L^{\infty}(m)$ is not order continuous we still can prove that $m_{T}$ is a (countably additive) vector measure. Consider a measurable partition $\left\{A_{i}\right\}_{i=1}^{\infty}$ of the measurable set $A$. Then for each $\eta$ we have that $\lim _{n} \int_{\cup_{i=1}^{n} A_{i}} g_{\eta} d m=\int_{A} g_{\eta} d m$. Notice that the 
domination (2) also holds in this case, and so for each $n \in \mathbb{N}$,

$$
\left\langle T\left(\chi_{A \backslash \cup_{i=1}^{n} A_{i}}\right), x^{\prime}\right\rangle=\lim _{\eta}\left\langle\int_{A \backslash \cup_{i=1}^{n} A_{i}} g_{\eta} d m, x^{\prime}\right\rangle \leq\left|\left\langle m, x^{\prime}\right\rangle\right|\left(A \backslash \cup_{i=1}^{n} A_{i}\right) .
$$

But each measure $\left|\left\langle m, x^{\prime}\right\rangle\right|$ is countably additive, so

$$
\lim _{n}\left\langle T\left(\chi_{A \backslash \cup_{i=1}^{n} A_{i}}\right), x^{\prime}\right\rangle=0
$$

what means that the set function $m_{T}$ defines a weakly countably additive vector measure. The Orlicz-Pettis Theorem ([3, Cor.4]) proves that $m_{T}$ is countably additive, and as we said it is also scalarly dominated by $m$.

To finish the proof for both cases (i) and (ii), it is enough to apply the Radon-Nikodym Theorem for scalarly dominated measures ([21, Th.1], see also [2, Th.3.1]). It gives that there is a function $g \in L^{\infty}(m)$ such that $T\left(\chi_{A}\right)=\int_{A} g d m, A \in \Sigma$. Since simple functions are dense in $L^{p^{\prime}}(m)$-including the case $p=\infty$ - we obtain that $T(f)=\int_{\Omega} g f d m$,

for all $f \in L^{p^{\prime}}(m)$. So, the unit ball of $L^{\infty}(m)$ is closed for the $\tau_{m^{-}}$ topology, and then compact.

Remark 11. As $\tau_{m}$ coincides with the weak ${ }^{*}$ topology whenever $m$ is a scalar measure, then the above result can be considered as an extension of Alaoglu's Theorem to vector measures with relatively compact range.

\section{Applications}

In this section, we present some applications regarding compactness of the unit ball of $L^{p}(m)$. In what follows we show a general description of the natural topologies on the space $L^{p}(m)$ and the links that can be established between them under the assumption of $\tau_{m}$-compactness of the unit ball. In order to do it, let us introduce the following notion. A Saks space is a triple $(E,\|\|,. \tau)$ where $(E,\|\|$.$) is a normed space and$ 
$\tau$ is a locally convex topology on $E$ so that $B_{E}$, the closed unit ball of $(E,\|\|$.$) , is \tau$-bounded and $\tau$-closed.

This definition was given by Cooper using the terminology introduced earlier by Orlicz for a related, although different, concept (see [1, Notes I.5] for details). For the general theory of Saks spaces we refer to [1]. The importance of Saks spaces lies in the existence of a mixed locally convex topology $\gamma[\|\|,. \tau]$ on $E$, which is the finest linear topology on $E$ that coincides with $\tau$ on the $\|\cdot\|$-bounded sets. The mixed topology $\gamma[\|\|,. \tau]$ is defined as follows: if $\mathcal{U}:=\left(U_{n}\right)_{n}$ is a sequence of absolutely convex 0 -neighbourhoods for $\tau$, the family formed by the sets

$$
\gamma(\mathcal{U}):=\cup_{n=1}^{\infty}\left(U_{1} \cap B_{E}+U_{2} \cap 2 B_{E}+\cdots+U_{n} \cap n B_{E}\right)
$$

when varying $\mathcal{U}$, is a basis of 0 -neighbourhoods for $\gamma[\|\|,. \tau]$.

Proposition 12. Let $1<p \leq \infty$. The spaces $\left(L^{p}(m),\|\cdot\|_{L^{p}(m)}, \tau_{m}\right)$ and $\left(L^{p}(m),\|\cdot\|_{L^{p}(m)}, \tau_{w, m}\right)$ are Saks spaces.

Proof. By [28, Proposition 12] $B_{L^{p}(m)}$ is $\tau_{w, m^{-}}$-closed and hence, $\tau_{m^{-}}$ closed.

Therefore we can consider the mixed topologies $\gamma_{m}:=\gamma\left[\|\cdot\|_{L^{p}(m)}, \tau_{m}\right]$ and $\gamma_{w, m}:=\gamma\left[\|\cdot\|_{L^{p}(m)}, \tau_{w, m}\right]$. So defined, $\gamma_{m}$ and $\gamma_{w, m}$ are the finest linear topologies that coincide with $\tau_{m}$ and $\tau_{w, m}$ respectively on $B_{L p(m)}$.

Proposition 13. Let $1<p \leq \infty$. If $B_{L^{p}(m)}$ is $\tau_{m}$-compact, then $\gamma_{w, m}=\gamma_{m}$ on bounded sets.

Proof. It follows from Proposition 1 and [9].

In case that $\gamma_{w, m}=\gamma_{m}$, we will just call such topology $\gamma$.

Proposition 14. Let $1<p<\infty$. If $L^{p}(m)$ is reflexive, then $\gamma_{w, m}$ is the topology of uniform convergence on compact subsets of $L^{p}(m)^{*}$. 
Proof. By [8, Theorem 2.1.7] $\tau_{w, m}$ coincides with the weak topology on $B_{L^{p}(m)}$. Since $L^{p}(m)$ is reflexive, $B_{L^{p}(m)}$ is weakly compact and hence $\tau_{w, m}$ compact. By [1, Corollary I.4.2], $\gamma_{w, m}$ is the finest topology on $L^{p}(m)$ which agrees with $\tau_{w, m}$ and hence with the weak topology on $B_{L^{p}(m)}$. As the weak and the weak* topologies coincide on $L^{p}(m)$, the Banach-Dieudonné Theorem yields the result.

From Propositions 1, 13 and 14 we get the following result.

Corollary 15. Let $1<p<\infty$. If $B_{L^{p}(m)}$ is $\tau_{m}$-compact, then $\gamma$ is the topology of uniform convergence on compact subsets of $L^{p}(m)^{*}$.

Proposition 16. Let $1<p<\infty$. If $B_{L^{p}(m)}$ is $\tau_{m}$-compact, then $\gamma$ is generated by all seminorms

$$
p(f):=\sup _{j} a_{j} \max _{k \leq j}\left\|\int_{\Omega} f h_{k} d m\right\|_{X}
$$

where $\left(a_{j}\right)_{j}$ varies over all sequences of positive numbers decreasing to 0 , and $\left(h_{j}\right)_{j}$ varies over all sequences in $B_{L^{p^{\prime}}(m)}$.

Proof. For each finite subset $H$ of $L^{p^{\prime}}(m)$, define

$$
p_{H}(f):=\max \left\{\left\|\int_{\Omega} f h d m\right\|_{X}: h \in H\right\} .
$$

Given an arbitrary $\epsilon>0$,

$$
\left\{f \in L^{p}(m): p_{H}(f)<\epsilon\right\}=\cap_{h \in H}\left\{f \in L^{p}(m):\left\|\int_{\Omega} f h d m\right\|_{X}<\epsilon\right\} .
$$

Then it is clear that $p_{H}$ is a continuous seminorm for $\tau_{m}$. Hence, when varying $H$ over all finite subsets of $L^{p^{\prime}}(m),\left\{p_{H}\right\}$ defines a family $S$ of continuous seminorms. Since $p_{\cup_{i=1}^{n} H_{i}}(f)=\max _{1 \leq i \leq n} p_{H_{i}}(f)$, the family $S$ is closed for finite suprema. Besides, since

$$
\begin{aligned}
\|f\|_{L^{p}(m)}= & \sup \left\{\left|\int_{\Omega} f h d\left\langle m, x^{*}\right\rangle\right|: h \in B_{L^{p^{\prime}}(m)}, x^{*} \in B_{E^{*}}\right\} \\
& =\sup \left\{\left\|\int_{\Omega} f h d m\right\|_{X}: h \in B_{L^{p^{\prime}}(m)}\right\}
\end{aligned}
$$


for all $f \in L^{p}(m)$, we obtain

$$
\|f\|_{L^{p}(m)}=\sup \left\{p_{H}(f): p_{H} \in S, H \subseteq B_{L^{p^{\prime}(m)}}\right\} .
$$

It follows from [1, Proposition I.4.5] that the seminorms $q$ given by $q(f)=\sup _{n} a_{n} p_{H_{n}}(f)$ generates the mixed topology $\gamma_{m}$ when $\left(a_{n}\right)_{n}$ varies over all sequences of positive numbers decreasing to 0 , and $\left(p_{H_{n}}\right)_{n}$ over all sequences in $S$. The result follows now easily.

\section{REFERENCES}

[1] J. B. Cooper, Saks spaces and Applications to Functional Analysis. NorthHolland Mathematics Studies, 28. North-Holland, Amsterdam, 1978.

[2] O. Delgado and E.A. Sánchez Pérez, Strong factorizations between couples of operators on Banach function spaces, J. Convex Anal. 20, 2 (2013), to appear.

[3] J. Diestel and J. J. Uhl, Vector Measures, Math. Surveys, vol. 15, Amer. Math. Soc., Providence, RI, 1977.

[4] D. Dubin, J. Kiukas, J.-P. Pellonpää and K. Ylinen, Operator integrals and sesquilinear forms. Preprint. arXiv:1303.1770.

[5] G. P. Curbera, Banach space properties of $L^{1}$ of a vector measure, Proc. Amer. Math. Soc. 123 (1995), 3797-3806.

[6] A. Fernández, F. Mayoral, F. Naranjo, C. Sáez and E.A. Sánchez-Pérez, Spaces of p-integrable functions with respect to a vector measure, Positivity 10 (2006), $1-16$.

[7] I. Ferrando and E. A. Sánchez Pérez, Tensor product representation of the (pre)dual of the Lp-space of a vector measure, J. Austr. Math. Soc. 87 (2009), 211-225.

[8] I. Ferrando, Duality in spaces of $p$-integrable functions with respect to a vector measure, Ph. D. Thesis. Universidad Politécnica de Valencia, 2009.

[9] I. Ferrando and J. Rodríguez, The weak topology on $L^{p}$ of a vector measure, Topology Appl., 155,13 (2008) 1439-1444.

[10] F. Galaz-Fontes, The dual space of $L^{p}$ of a vector measure, Positivity 14,4 (2010), 715-729.

[11] L. M. García-Raffi and B. Jefferies, An application of bilinear integration to quantum scattering. J. Math. Anal. Appl. (2014), http://dx.doi.org/10.1016/ j.jmaa.2014.01.055

[12] L. M. García-Raffi, E. Jiménez Fernández and E. Sánchez Pérez, A non-linear approach to signal processing by means of vector measure orthogonal functions. Publ. Res. Inst. Math. Sci. 49 (2013), 2, 241-269.

[13] L. M. García-Raffi and E. A. Sánchez-Pérez, Compactness properties of bounded subsets of spaces of vector measure integrable functions and factorization of operators, App. Gen. Top. 6,2 (2005), 135-142.

[14] L. M. García Raffi, E. A. Sánchez Pérez and J. V. Sánchez Pérez, Commutative sequences of integrable functions and best approximation with respect to the 
weighted vector measure distance. Integral Equations Operator Theory 54, 4 (2006), 495-510.

[15] B. Jefferies, Evolution processes and the Feynman-Kac formula. Mathematics and its Applications, 353. Kluwer Academic Publishers Group, Dordrecht, 1996.

[16] E, Jiménez-Fernández, Orthogonal systems in $L^{2}$ spaces of a vector measure. Turk. J. Math., 37 (2013), 981-992.

[17] I. Kluvánek; G. Knowles, Vector measures and control systems. North-Holland Mathematics Studies, 20. North-Holland, Amsterdam, 1976.

[18] D. R. Lewis, Integration with respect to vector measures, Pacific J. Math., 33 (1970), 157-165.

[19] D.R. Lewis, On integrability and summability in vector spaces , Illinois J. Math., 16,2 (1972), 294-307.

[20] J. Lindenstrauss and L. Tzafriri, Classical Banach Spaces, II, Springer, Berlin, 1996.

[21] K. Musial, A Radon-Nikodým theorem for the Bartle-Dunford-Schwartz integral, Atti Sem. Mat. Fis. Univ. Modena XLI (1993), 227-233.

[22] Ng, K., On a theorem of Dixmier, Math. Scand., 29 (1971), 279-280.

[23] S. Okada, and W. J. Ricker, The range of the integration map of a vector measure, Arch. Math. 64 (1995), 512-522.

[24] S. Okada, W.J. Ricker, and L. Rodríguez-Piazza, Compactness of the integration operator associated with a vector measure, Studia Math. 150,2 (2002), 133-149.

[25] S. Okada, W.J. Ricker, and L. Rodríguez-Piazza, Operator ideal properties of vector measures with finite variation, Studia Math. 250,3 (2011), 215-249.

[26] S. Okada, W.J. Ricker and E.A. Sánchez Pérez, Optimal domain and integral extension of operators acting in function spaces. Operator Theory: Advances and Applications, 180. Birkhäuser Verlag, Basel, 2008.

[27] P. Rueda, and E. A. Sánchez Pérez, Factorization theorems for homogeneous maps on Banach function spaces and approximation of compact operators. Mediterranean J. Math. To appear.

[28] E. A. Sánchez Pérez, Compactness arguments for spaces of p-integrable functions with respect to a vector measure and factorization of operators through Lebesgue-Bochner spaces, Illinois J. Math. 45, 3 (2001), 907-923.

[29] E.A. Sánchez Pérez, Vector measure duality and tensor product representations of $L_{p}$-spaces of vector measures, Proc. Am. Math. Soc. 132,11 (2004), 3319 3326 .

[Pilar Rueda] Departamento de Análisis Matemático, Universidad de Valencia, 46100 Burjassot - Valencia, Spain, e-mail: pilar.rueda@uv.es [Enrique A. Sánchez Pérez] Instituto Universitario de Matemática Pura y Aplicada, Universidad Politècnica de València, Camino de Vera s/n, 46022 Valencia, Spain, e-mail: easancpe@mat.upv.es 